\title{
EFFECTIVE VISCOSITY OF DISPERSIONS APPROACHED BY A STATISTICAL CONTINUUM METHOD
}

\author{
J. MELLEMA and M.W.M. WILLEMSE \\ Rheolog, Group, Department of Applied Physics, Twente University of Technology, Enschede, \\ The Netherlands
}

Received 1 March 1983

Manuscript received in final form 16 June 1983

\begin{abstract}
The problem of the determination of the effective viscosity of disperse systems (emulsions, suspensions) is considered. On the basis of the formal solution of the equations governing creeping flow in a statistically homogeneous dispersion, the effective viscosity is expressed in a series expansion in terms of correlation functions. The contribution of the interfacial tension to the effective viscosity is also considered and finally bounds for the effective viscosity are indicated.
\end{abstract}

\section{Introduction}

The rheology of dispersions is a growing field of interest (Mewis and Spaull') and Herczyński and Pieńkowska $\left.{ }^{2}\right)$ ). Dispersions can be subdivided into two classes, namely suspensions and emulsions. Suspensions are defined as fine rigid particles suspended in a fluid. In emulsions (un)deformable fluid particles are immersed in another fluid. The general problem to be solved is the description of the flow of dispersions including a relationship between microscopic and macroscopic behaviour. The key to this problem is the formulation of a constitutive equation. A particular member of this family of problems is the determination of the Newtonian part of the constitutive equation. This is the subject of the present paper.

First of all one has to define the dispersion and flow characteristics. In this paper the dispersion consists of two incompressible Newtonian fluids, with viscosities $\eta_{1}$ and $\eta_{2}$, subjected to a steady motion. The influence of inertia forces and Brownian motion is neglected. The contact area between the fluids is characterized with a constant interfacial tension $\gamma$. The dispersion is considered as statistically homogeneous and isotropic. For $\eta_{2} \rightarrow \infty$ the dispersion can be regarded as a suspension. In section 2 the starting points and basic assumptions are discussed more extensively.

The first investigation of such a system was carried out for its most simple manifestation. It was presented by Einstein ${ }^{3}$ ), who calculated the effective viscosity $\eta$ * of a suspension of rigid spheres with negligible hydrodynamic interactions; in

0378-4371/83/0000-0000/\$03.00@1983 North-Holland 
first order of the volume fraction $\phi$ he found

$$
\eta^{*}=\eta_{1}\left(1+\frac{5}{2} \phi\right)
$$

where $\eta_{1}$ is the viscosity of the fluid surrounding the particles. It lasted till 1932 that this result was generalized by Taylor $^{4}$ ) for an emulsion of almost spherical particles $(\gamma \rightarrow \infty)$ of a Newtonian fluid with viscosity $\eta_{2}$, neutrally buoyant in a Newtonian fluid with viscosity $\eta_{1}$,

$$
\eta^{*}=\eta_{1}\left(1+\frac{2 \eta_{1}+5 \eta_{2}}{2\left(\eta_{1}+\eta_{2}\right)} \phi\right)
$$

Both results are easy to obtain, but a plausible expectation in those times that the problem of the formulation of the rheological properties of dispersions might be solved gradually with increasing detail has not been realized. Only the calculation of the effective viscosity for rigid spheres with two-particle hydrodynamic interactions resulting in

$$
\eta^{*}=\eta_{1}\left(1+\frac{5}{2} \phi+(7.6 \pm 0.8) \phi^{2}\right)
$$

presented by Batchelor and Green $^{5}$ ), is a step in this direction. The generalization of Taylor's result to two-particle interactions is absent, as is the calculation of the influence of multiparticle interactions on $\eta^{*}$.

The difficulties that are to be encountered can be imagined by setting up a hierarchy of flow equations in which the sequence is determined by the number $N$ of the $N$-particle conditional average of a traction integrated over the surface of one particle, keeping the position of $N-1$ other particles constant. The use of a Heaviside-like function and ensemble averaging makes it easy to arrive at this hierarchy (Lundgren $\left.{ }^{6}\right)$ ). This hierarchy clarifies the significance and limitations of the solution for one particle in a flow, for two particles in a flow, etc., and it demonstrates the ambivalence of cell models.

In this article the hierarchy approach is not used, but the possibilities of a statistical continuum method $\left(\right.$ Beran $\left.^{7}\right)$ ) are investigated. The fact is that the Heaviside-like function can also be used for a dispersion of two Newtonian fluids to indicate the viscosity of the different fluids. Thus the viscosity can be represented as a generalized position-dependent function. Separating this function in a position-dependent and -independent part offers the opportunity to solve the equations of motion by means of Oseen-operators. Then the averaged deviatoric part of the stress tensor for some particular dispersions can be developed in a series proportional to the averaged rate-of-strain tensor and this gives an expression for the effective viscosity. The series can be subdivided into a part that is texture-independent and another part that is texture-dependent. The textureindependent part represents the so-called effective-medium viscosity. By taking $\eta_{2} \rightarrow \infty$ this effective-medium viscosity equals the viscosity of a suspension of rigid 
spheres found by Lundgren ${ }^{6}$ ), by an ad hoc closure of the mentioned hierarchy of flow equations. Another result is the deduction of bounds for the viscosity, though already found in another way by $\mathrm{Hashin}^{8}$ ). The presented statistical continuum approach is conceptually founded in work on electrical conductivity of polycrystalline materials (Willemse ${ }^{9}$ ) and Willemse and Caspers ${ }^{10}$ )). Its feasibility for dispersions is investigated in the present paper.

In the discussion the results are confronted with the results of two partially competitive lines in the research of the rheological properties of dispersions. The first one (Nagatani ${ }^{11}$ ), Bedeaux et al. $\left.{ }^{12}\right)$ ) is akin to the presented method, because in this approach the flow equations are also solved formally by the use of Oseen-operators. The difference, however, is that first the flow equations are Fourier-transformed. The second line $\left(\right.$ Hashin $\left.^{8}\right)$, Keller et al. ${ }^{13}$ ) and Batchelor $\left.{ }^{14}\right)$ ) tries to find bounds for the viscosity by using variational techniques and these results are comparable with the bounds found by the presented method.

\section{Basic equations governing creeping flow}

The state of a moving fluid is determined by five quantities, viz. the three components of the velocity $\boldsymbol{u}$, the pressure $p$, and the density $\rho$ (Landau and Lifshitz $\left.{ }^{15}\right)$ ). For the description of the motion of a dispersion in which the fluid particles are neutrally buoyant only four quantities are needed, since the density $\rho$ is assumed to be constant throughout. Consequently, the hydrodynamics of a dispersion is sufficiently characterized by four equations of motion. These four equations may be derived from two principles: the conservation of mass and of momentum, which read respectively

$$
\begin{aligned}
& \frac{\partial}{\partial t} \rho+\frac{\partial}{\partial x_{i}}\left(\rho u_{i}\right)=0, \\
& \rho \frac{\partial u_{i}}{\partial t}+\rho u_{j} \frac{\partial u_{i}}{\partial x_{j}}=\frac{\partial}{\partial x_{j}} \Sigma_{i j},
\end{aligned}
$$

where $\Sigma_{i j}$ represents a component of the stress tensor and where a Cartesian coordinate system is employed and Einstein's summation convention is utilized.

In order to make this set of equations more tractable and, what is more, linear a few simplifying assumptions will be made.

The non-linear term $\rho(\boldsymbol{u} \cdot \boldsymbol{\nabla}) \boldsymbol{u}$ may be neglected with respect to the term on the right-hand side of (2.2) if the Reynolds number $\operatorname{Re}=l u \rho / \eta \ll 1$, where $\eta$ is the coefficient of viscosity. The Reynolds number of a type of flow indicates the ratio of the inertia forces and the viscous forces. The quantities $l, u, \rho$, and $\eta$ appearing in the expression for the Reynolds number, are parameters characterizing the fluid 
and its kinematic behaviour and are typical for the type of flow. In the case of a dispersion consisting of fluid particles with dimension $a$, the characteristic length $l$ equals $a$. If the type of flow is taken to be extensional, i.e. $u_{i}=\alpha_{i j} x_{j}$, with $\alpha$ a symmetric, traceless and constant tensor, the condition for the Reynolds number in the neighbourhood of the fluid particles results in $\alpha a^{2} \rho / \eta \ll 1$, with $\alpha=\sqrt{\alpha_{i j} \alpha_{i j}}$. This condition must hold in the inner region as well as in the outer region of the fluid particles. Obviously, this condition will be violated in the distant outer region. However, this difficulty will not be regarded here and it will be assumed that $\rho(\boldsymbol{u} \cdot \nabla) \boldsymbol{u}=0$ everywhere.

For steady extensional flow of the dispersion on a macroscopic scale the term $\rho\left(\partial u_{i} / \partial t\right)$ in (2.2) may be neglected on a microscopic scale if the volume fraction $\phi$ of the fluid particles is small as compared to unity. In that case the motion of every fluid particle is affine and the quantity $\partial u_{i} / \partial t$ may be estimated to be of the same order of magnitude as the term $u_{j}\left(\partial u_{i} / \partial x_{j}\right)$, because every fluid particle may be thought of as being embedded in an unperturbed fluid flow. Without further justification it is assumed in the remainder that $\partial u_{i} / \partial t$ vanishes everywhere.

The effect of Brownian motion is not taken into account in this paper. For spherical particles with radius $a$ the mean squared distance traversed in a time $t$ is given by $\overline{r^{2}}=6 D t$, where the diffusion constant $D$ equals $D=k T / 6 \pi \eta a$. From these expressions it is seen that the velocity as a consequence of Brownian movement depends on $D$ and that this contribution to the velocity of the particles becomes smaller if the temperature $T$ is lowered or if the size of the particles is increased. The only essential aspect of Brownian motion regarding the effective viscosity of a dispersion is its influence on the probability distribution function of the arrangement of fluid particles in the continuous phase. According to Russel ${ }^{16}$ ), the effect of Brownian motion is dominated by hydrodynamic forces on the condition that the translational Péclet number $\mathrm{Pe}_{\mathrm{t}}=\eta a^{3} \alpha / k T \gg 1$. Hence, the latter and former condition restrain the flow strength by $k T / \eta a^{3} \ll \alpha \ll \eta / a^{2} \rho$, for the subsequent results to have a chance of validity.

In order to be able to solve the equations of motion (2.1) and (2.2) one needs a constitutive equation, connecting the fields $\boldsymbol{u}$ and $\boldsymbol{\Sigma}$. The dispersion consists of a continuous phase containing fluid particles, both of which are assumed to be Newtonian. The transition of one phase to the other is treated as an infinitesimally thin and non-material interface, which is characterized with a constant interfacial tension $\gamma$.

In a Newtonian fluid the stress tensor $\Sigma$ only depends linearly on the rate-of-strain tensor $\boldsymbol{D}$, defined by

$$
D_{i j}=\frac{1}{2}\left(\frac{\partial u_{i}}{\partial x_{j}}+\frac{\partial u_{j}}{\partial x_{i}}\right),
$$

and by assuming the interfacial tension tensor $\Gamma$ to be two-dimensionally 
isotropic, the constitutive equation reads (see appendix A)

$$
\Sigma_{i j}=\sigma_{i j}+\Gamma_{i j}=-p \delta_{i j}+2 \eta D_{i j}+\gamma\left(\delta_{i j}-n_{i} n_{j}\right) \delta\left(\boldsymbol{r}-\boldsymbol{r}_{0}\right) .
$$

Here the unit normal vector $\boldsymbol{n}$ points out of the fluid particle, the boundary of which is described by $\boldsymbol{r}_{0}$ occurring in the argument of the Dirac delta function $\delta\left(\boldsymbol{r}-\boldsymbol{r}_{0}\right)$.

Of course, the constitutive equation of the interface in (2.4) may be extended with, for instance, a viscous term (Oosterbroek and Mellema $\left.{ }^{17}\right)$ ), but here a nonmaterial interface is chosen. Note that the interfacial tension contains only tangential components and no normal component. This is easily checked by contraction of the tensor $U_{\mathrm{II}}$, with components $\delta_{i j}-n_{i} n_{j}$, with the unit normal $\boldsymbol{n}$.

The spatial distribution of two immiscible phases in the dispersion can be described in an elegant way by the indicatrix $\xi$, defined by

$$
\xi(\boldsymbol{r})= \begin{cases}0, & \boldsymbol{r} \text { in solvent } \\ 1, & \boldsymbol{r} \text { in fluid particle }\end{cases}
$$

which enables one to mark the interface by

$$
\frac{\partial \xi}{\partial x_{i}}=-n_{i} \delta\left(\boldsymbol{r}-\boldsymbol{r}_{0}\right)
$$

The viscosity is then given by

$$
\eta(\boldsymbol{r})=\eta_{1}+\left(\eta_{2}-\eta_{1}\right) \xi(\boldsymbol{r})
$$

with $\eta_{1}$ the viscosity of the solvent and $\eta_{2}$ the viscosity of the fluid particles.

Summarizing, the conservation equations (2.1) and (2.2) become in the continuous phases, respectively,

$$
\frac{\partial u_{i}}{\partial x_{i}}=0
$$

and

$$
\eta \frac{\partial^{2} u_{i}}{\partial x_{j}^{2}}=\frac{\partial p}{\partial x_{i}}
$$

while at the interface of the two fluids they become respectively

$$
u_{i} n_{i}=U_{i} n_{i}
$$

and

$$
\left[\sigma_{i j}\right] n_{j}=\gamma n_{i} \frac{\partial n_{j}}{\partial x_{j}}-\frac{\partial \gamma}{\partial x_{j}}\left(\delta_{i j}-n_{i} n_{j}\right)
$$

where $U$ denotes the velocity of the interface, which has only a normal component, 
and where the square bracket $\left[\sigma_{i j}\right]$ means the magnitude of the discontinuity at the interface (see appendix A).

Since $\gamma$ is assumed to be a constant throughout the interface, (2.11) reduces to

$$
\left[\sigma_{i j}\right] n_{j}=\gamma n_{i} \frac{\partial n_{j}}{\partial x_{j}}
$$

\section{Definition of the effective viscosity}

In defining the effective viscosity $\eta^{*}$ of a dispersion the basic assumption is that the dispersion behaves macroscopically as a Newtonian fluid. From this assumption it follows that the rate-of-strain tensor $\boldsymbol{D}$ of the main flow must be translationally invariant to effect a homogeneous (random) distribution of particles.

For the definition one may use two different starting points, viz. the constitutive equation and the energy dissipation. The constitutive equation relates the macroscopic quantities $\left\langle\Sigma_{i j}\right\rangle$ and $\left\langle D_{i j}\right\rangle$ as follows:

$$
\left\langle\Sigma_{i j}\right\rangle-\frac{1}{3}\left\langle\Sigma_{k k}\right\rangle \delta_{i j} \equiv 2 \eta^{*}\left\langle D_{i j}\right\rangle \text {. }
$$

Here the bracket denotes volume averaging,

$$
\langle A\rangle \equiv \lim _{V \rightarrow \infty} \frac{1}{V} \int_{V} A \mathrm{~d} V,
$$

where $V$ is the volume over which the averaging is performed. Note that the definition implies that $\langle A\rangle$ is translationally invariant. Another way of averaging is ensemble averaging accomplished by averaging the statistical quantity over an admissible ensemble of realizations, weighing thus with a probability density of the realizations. The advantage of volume averaging is the absence of boundary effects (Herczyński and Pieńkowska ${ }^{2}$ )).

The definition making use of the energy dissipation, expresses that the total dissipation in the dispersion equals the dissipation of a microscopically homogeneous Newtonian fluid obeying the same boundary conditions:

$$
\left\langle\sum_{i j} D_{i j}\right\rangle \equiv 2 \dot{\eta}^{*}\left\langle D_{i j}\right\rangle\left\langle D_{i j}\right\rangle \text {. }
$$

In (3.1) it is assumed that the deviator of the averaged surface tension tensor is linearly related to the averaged rate-of-strain tensor $\langle\boldsymbol{D}\rangle$. In general, the two tensors would be related like

$$
\left\langle\gamma\left(\frac{1}{3} \delta_{i j}-n_{i} n_{j}\right) \delta\left(\boldsymbol{r}-\boldsymbol{r}_{0}\right)\right\rangle=f\left(\left\langle D_{i j}\right\rangle\right),
$$

where $f$ is some appropriate function of $\left\langle D_{i j}\right\rangle$, but the assumption of linearity 
leads to

$$
\left\langle\gamma\left(\frac{1}{3} \dot{\delta}_{i j}-n_{i} n_{i}\right) \delta\left(\boldsymbol{r}-\boldsymbol{r}_{0}\right)\right\rangle=c\left\langle D_{i j}\right\rangle .
$$

with c constant.

The step from (3.4) to (3.5) can be seen as a truncation of a Taylor series expansion that becomes more valid with decreasing $\left\langle D_{i j}\right\rangle$. From (3.5) it is seen that the averaging of the deformation of the fluid particles comes to averaging of the tensor $\boldsymbol{n} \boldsymbol{n}$, having the same principal directions as the $\langle\boldsymbol{D}\rangle$ tensor, and that the contribution of the surface tension tensor to the effective viscosity $\eta^{*}$ depends on the combination of the magnitude of the surface tension and the resulting deformation. It is hard to say under what conditions regarding the values of $\gamma$ and the ratio $\eta_{2} / \eta_{1}$ eq. (3.5) can be true. It is felt that for sufficiently large values of $\gamma$ and constant value of $\langle\boldsymbol{D}\rangle$ eq. (3.5) is probably true due to resulting small deformations of the spheres.

The average of the stress tensor $\Sigma$ appearing in (3.1) may be replaced by (see appendix B)

$$
\left\langle\Sigma_{i i}\right\rangle=\left\langle\frac{\partial}{\partial x_{k}}\left(\sigma_{i k}, x_{j}\right)\right\rangle
$$

so that the first definition (3.1) could also read

$$
\left\langle\frac{\partial}{\partial x_{k}}\left(\sigma_{i k} x_{j}\right)\right\rangle-\frac{1}{3}\left\langle\frac{\partial}{\partial x_{k}}\left(\sigma_{i k} x_{i}\right)\right\rangle \delta_{i j} \equiv 2 \eta^{*}\left\langle D_{i i}\right\rangle .
$$

By defining the effective viscosity $\eta^{*}$ in this way it seems that it does not depend on the averaged surface tension tensor, a problem that was extensively discussed by Batchelor ${ }^{18}$ ).

The second definition of the effective viscosity, using the dissipation, can be simplified to

$$
\begin{aligned}
2 \eta^{*}\left\langle D_{i j}\right\rangle\left\langle D_{i j}\right\rangle & =\left\langle\sum_{i j} D_{i j}\right\rangle \\
& =\left\langle\left(\sum_{i j}-\frac{1}{3} \sum_{k k} \delta_{i j}\right) D_{i j}\right\rangle \\
& =\left\langle\left(2 \eta D_{i j}+\gamma\left(\frac{1}{3} \delta_{i i}-n_{i} n_{j}\right) \delta\left(\boldsymbol{r}-\boldsymbol{r}_{i}\right)\right) D_{i j}\right\rangle \\
& =\left\langle 2 \eta D_{i j} D_{i j}\right\rangle .
\end{aligned}
$$

The last step is possible since the steady main flow constitutes locally a stationary stochastic process in which the time-derivative of the surface energy $\gamma S$, being

$$
\frac{\mathrm{d}}{\mathrm{d} t}(\gamma S)=-\left\langle\gamma n_{i} n_{j} D_{i j} \delta\left(\boldsymbol{r}-\boldsymbol{r}_{i j}\right)\right\rangle
$$

with $S$ the total area of the surface of all fluid particles, vanishes (see Rosenkilde $\left.{ }^{19}\right)$ ). 
The fact that no effective storage of elastic energy results is satisfactory since it was assumed that the dispersion behaves macroscopically Newtonian.

The consistency, or rather the requirement of consistency, of the two definitions of the effective viscosity $\eta^{*}$ may be used for obtaining bounds for $\eta^{*}$. To do so the rate-of-strain tensor $D$ is subdivided into the average value and its fluctuations,

$$
D_{i j}=\left\langle D_{i j}\right\rangle+D_{i j}^{\prime} .
$$

Note that the average value of the fluctuations vanishes: $\left\langle D_{i j}^{\prime}\right\rangle=0$. The two definitions given in (3.1) and (3.8) being compared,

$$
\begin{aligned}
& \eta^{*}\left\langle D_{i j}\right\rangle=\left\langle\eta D_{i j}\right\rangle+\frac{1}{2}\left\langle\gamma\left(\frac{1}{3} \delta_{i j}-n_{i} n_{j}\right) \delta\left(\boldsymbol{r}-\boldsymbol{r}_{0}\right)\right\rangle, \\
& \eta^{*}\left\langle D_{i j}\right\rangle\left\langle D_{i j}\right\rangle=\left\langle\eta D_{i j} D_{i j}\right\rangle,
\end{aligned}
$$

and (3.11) being multiplied by $\left\langle D_{i j}\right\rangle$, it holds

$$
\left\langle\eta D_{i j}\right\rangle\left\langle D_{i j}\right\rangle+\frac{1}{2}\left\langle\gamma\left(\frac{1}{3} \delta_{i j}-n_{i} n_{j}\right) \delta\left(\boldsymbol{r}-\boldsymbol{r}_{0}\right)\right\rangle\left\langle D_{i j}\right\rangle=\left\langle\eta D_{i j} D_{i j}\right\rangle .
$$

Applying (3.10) in this equation leads to

$$
\left\langle\eta D_{i j}^{\prime}\right\rangle\left\langle D_{i j}\right\rangle+\left\langle\eta D_{i j}^{\prime} D_{i j}^{\prime}\right\rangle-\frac{1}{2}\left\langle\gamma\left(\frac{1}{3} \delta_{i j}-n_{i} n_{j}\right) \delta\left(\boldsymbol{r}-\boldsymbol{r}_{0}\right)\right\rangle\left\langle D_{i j}\right\rangle=0,
$$

and finally insertion of this result in equation (3.12) gives

$$
\begin{aligned}
\eta^{*}\left\langle D_{i j}\right\rangle\left\langle D_{i j}\right\rangle= & \langle\eta\rangle\left\langle D_{i j}\right\rangle\left\langle D_{i j}\right\rangle-\left\langle\eta D_{i j}^{\prime} D_{i j}^{\prime}\right\rangle \\
& +\frac{1}{2}\left\langle\gamma\left(\frac{1}{3} \delta_{i j}-n_{i} n_{j}\right) \delta\left(\boldsymbol{r}-\boldsymbol{r}_{0}\right)\right\rangle\left\langle D_{i j}\right\rangle .
\end{aligned}
$$

Since $\left\langle\eta D_{i j}^{\prime} D_{i j}^{\prime}\right\rangle$ is positive semi-definite, it follows:

$$
\eta^{*} \leqslant\langle\eta\rangle+\frac{c}{2}
$$

As $\gamma$ is assumed to be constant, only in two cases it is easy to obtain the simple result

$$
\eta^{*} \leqslant\langle\eta\rangle
$$

namely for $\gamma=0$ and for spherical particles with $\gamma \neq 0$.

The latter case, however, is not feasible in a flow as a consequence of the condition of force equilibrium at the particle interface given in eq. (2.12). For the remaining possibility, viz. $\gamma=0$, it is also possible to obtain the corresponding lower bound by working with the fluidity $1 / \eta$, being the inverse of the viscosity $\eta$,

$$
\langle 1 / \eta\rangle^{-1} \leqslant \eta^{*} \leqslant\langle\eta\rangle \text {. }
$$

These bounds were already found by $\mathrm{Hashin}^{8}$ ) and Batchelor ${ }^{14}$ ), but it must be emphasized that these bounds cannot be valid for immiscible fluids, because then 
$\gamma \neq 0$. The latter conclusion is in accord with the only result that is proved beyond any doubt (Keller et al. $\left.{ }^{13}\right)$ ), namely the so-called Einstein-Taylor formula, giving the effective viscosity $\eta^{*}$ in the first order of the volume fraction $\phi$ of the fluid particles (expression (1.2)).

\section{Formal solution of the flow fields}

The hydrodynamic problem of flow in a dispersion is given by the equations already given in section 2 :

$$
\begin{array}{ll}
\text { incompressible fluid: } & \frac{\partial u_{i}}{\partial x_{i}}=0, \\
\text { equilibrium of forces: } & \frac{\partial}{\partial x_{j}} \sigma_{i j}=\gamma n_{i} \frac{\partial n_{j}}{\partial x_{j}} \delta\left(\boldsymbol{r}-\boldsymbol{r}_{0}\right), \\
\text { constitutive equation: } & \sigma_{i j}=-p \delta_{i j}+2 \eta D_{i j}, \\
\text { viscosity: } & \eta(\boldsymbol{r})=\eta_{1}+\left(\eta_{2}-\eta_{1}\right) \xi(\boldsymbol{r}) .
\end{array}
$$

Now, in the last equation, the description of the spatial distribution of the viscosity, a new parameter $\eta_{0}$ is introduced aiming at the effective viscosity $\eta^{*}$ (see section 5),

$$
\eta(\boldsymbol{r})=\eta_{0}(1+q(\boldsymbol{r}))
$$

with

$$
q(\boldsymbol{r})=\frac{\eta(\boldsymbol{r})-\eta_{0}}{\eta_{0}}
$$

Inserting (4.3) in (4.2) and making use of (4.1), one arrives at the following differential equation:

$$
-\frac{\partial p}{\partial x_{i}}+\frac{\partial}{\partial x_{j}} \eta\left(\frac{\partial u_{i}}{\partial x_{j}}+\frac{\partial u_{j}}{\partial x_{i}}\right)=\gamma n_{i} \frac{\partial n_{j}}{\partial x_{j}} \delta\left(\boldsymbol{r}-\boldsymbol{r}_{0}\right)
$$

which becomes with the help of (4.5)

$$
-\frac{\partial p}{\partial x_{i}}+\eta_{0} \frac{\partial^{2} u_{i}}{\partial x_{j}^{2}}=\gamma n_{i} \frac{\partial n_{j}}{\partial x_{j}} \delta\left(\boldsymbol{r}-\boldsymbol{r}_{0}\right)-\eta_{0} \frac{\partial}{\partial x_{j}} q\left(\frac{\partial u_{i}}{\partial x_{j}}+\frac{\partial u_{j}}{\partial x_{i}}\right)
$$

By using the Oseen tensor and vector respectively this equation can be solved formally,

$$
\begin{aligned}
u_{i}\left(\boldsymbol{r}_{1}\right)= & u_{i}^{(0)}\left(\boldsymbol{r}_{1}\right)+\frac{1}{8 \pi \eta_{0}} \int_{V} \mathrm{~d} \boldsymbol{r}_{2}\left(\frac{\delta_{i j}}{r_{12}}+\frac{x_{12, i} x_{12, j}}{r_{12}^{3}}\right) \\
& \times\left\{-\gamma n_{j} \frac{\partial n_{k}}{\partial x_{2, k}} \delta\left(\boldsymbol{r}_{2}-\boldsymbol{r}_{0}\right)+\eta_{0} \frac{\partial}{\partial x_{2, k}} q\left(\frac{\partial u_{j}}{\partial x_{2, k}}+\frac{\partial u_{k}}{\partial x_{2, j}}\right)\right\}
\end{aligned}
$$


and

$$
\begin{aligned}
p\left(\boldsymbol{r}_{1}\right)= & p^{(0)}\left(\boldsymbol{r}_{1}\right)+\frac{-1}{4 \pi} \int_{V} \mathrm{~d} \boldsymbol{r}_{2} \frac{x_{12, j}}{r_{12}^{3}} \\
& \times\left\{-\gamma n_{j} \frac{\partial n_{k}}{\partial x_{2, k}} \delta\left(\boldsymbol{r}_{2}-\boldsymbol{r}_{0}\right)+\eta_{0} \frac{\partial}{\partial x_{2, k}} q\left(\frac{\partial u_{j}}{\partial x_{2, k}}+\frac{\partial u_{k}}{\partial x_{2, j}}\right)\right\},
\end{aligned}
$$

with $\boldsymbol{r}_{12} \equiv \boldsymbol{r}_{2}-\boldsymbol{r}_{1}$, and where $V$ designates the volume of the dispersion, eventually taken to be infinite; furthermore, $\boldsymbol{u}^{(0)}$ and $p^{(0)}$ are the additive solutions of eq. (4:7) in the case that the right-hand side equals zero.

By differentiating (4.8) with respect to $r_{1}$ one arrives at

$$
\begin{aligned}
D_{i j}\left(\boldsymbol{r}_{1}\right)= & D_{i j}^{(0)}\left(\boldsymbol{r}_{1}\right)+\frac{1}{8 \pi \eta_{0}} \int_{V} \mathrm{~d} \boldsymbol{r}_{2}\left(3 \frac{x_{12, i} x_{12, j} x_{12, k}}{r_{12}^{5}}-\delta_{i j} \frac{x_{12, k}}{r_{12}^{3}}\right) \\
& \times\left\{-\gamma n_{k} \frac{\partial n_{l}}{\partial x_{2, l}} \delta\left(\boldsymbol{r}_{2}-\boldsymbol{r}_{0}\right)+2 \eta_{0} \frac{\partial}{\partial x_{2, l}} q\left(\boldsymbol{r}_{2}\right) D_{k l}\left(\boldsymbol{r}_{2}\right)\right\},
\end{aligned}
$$

where $D_{i j}^{(0)}$ is the unperturbed rate-of-strain tensor, which was assumed to be translationally invariant.

Changing the set of independent variables $\boldsymbol{r}_{1}$ and $\boldsymbol{r}_{2}$ into $\boldsymbol{r}_{1}$ and $\boldsymbol{r}_{12}$, one can write (4.9) and (4.10) as

$$
\begin{aligned}
D_{i j}\left(\boldsymbol{r}_{1}\right)= & D_{i j}^{(0)}+\frac{1}{8 \pi \eta_{0}} \int_{V} \mathrm{~d} \boldsymbol{r}_{12}\left(3 \frac{x_{12, i} x_{12, j} x_{12, k}}{r_{12}^{5}}-\delta_{i j} \frac{x_{12, k}}{r_{12}^{3}}\right) \\
& \times\left\{-\gamma n_{k} \frac{\partial n_{l}}{\partial x_{12, l}} \delta\left(\boldsymbol{r}_{1}+\boldsymbol{r}_{12}-\boldsymbol{r}_{0}\right)+2 \eta_{0} \frac{\partial}{\partial x_{12, l}} q\left(\boldsymbol{r}_{1}+\boldsymbol{r}_{12}\right) D_{k l}\left(\boldsymbol{r}_{1}+\boldsymbol{r}_{12}\right)\right\}
\end{aligned}
$$

and

$$
\begin{aligned}
p\left(\boldsymbol{r}_{1}\right)= & p^{(0)}\left(\boldsymbol{r}_{1}\right)-\frac{1}{4 \pi} \int_{V} \mathrm{~d} \boldsymbol{r}_{12} \frac{x_{12, j}}{r_{12}^{3}} \\
& \times\left\{-\gamma n_{j} \frac{\partial n_{k}}{\partial x_{12, k}} \delta\left(\boldsymbol{r}_{1}+\boldsymbol{r}_{12}-\boldsymbol{r}_{0}\right)+2 \eta_{0} \frac{\partial}{\partial x_{12, k}} q\left(\boldsymbol{r}_{1}+\boldsymbol{r}_{12}\right) D_{j k}\left(\boldsymbol{r}_{1}+\boldsymbol{r}_{12}\right)\right\} .
\end{aligned}
$$

Eq. (4.11) can be written in a short-hand notation

$$
D_{i j}=D_{i j}^{(0)}+D_{i j}^{(\gamma)}+G_{i j k l} q D_{k l},
$$


where

$$
\begin{aligned}
D_{i j}^{(j)}\left(\boldsymbol{r}_{1}\right)= & \frac{1}{8 \pi \eta_{0}} \int_{i} \mathrm{~d} \boldsymbol{r}_{12}\left(3 \frac{x_{12, i} x_{12, j} x_{12, k}}{r_{12}^{5}}-\delta_{i j} \frac{x_{12, k}}{r_{12}^{3}}\right) \\
& \times\left\{-\gamma n_{k} \frac{\partial n_{1}}{\partial x_{12, i}} \delta\left(\boldsymbol{r}_{1}+\boldsymbol{r}_{12}-\boldsymbol{r}_{0}\right)\right\},
\end{aligned}
$$

and where the integral-operator $G_{i j k l}$ is defined by

$$
G_{i j k l}\left(\boldsymbol{r}_{1}\right)=\frac{1}{4 \pi} \int_{V} \mathrm{~d} \boldsymbol{r}_{12}\left(3 \frac{x_{12, i} x_{12, j} x_{12, k}}{r_{i 2}^{5}}-\delta_{i j} \frac{x_{12, k}}{r_{12}^{3}}\right) \frac{\imath}{\partial x_{12, l}} .
$$

Note that, since $D_{k l}$ is symmetrical with respect to the indices $k$ and $l$, only that part of $G_{i j k l}$ that is also symmetrical in the indices $k$ and $/$ operates effectively on $D_{k l}$, so that $G_{i j k l}$ may also be defined as follows:

$$
G_{i j k l}\left(\boldsymbol{r}_{1}\right)=\frac{1}{8 \pi} \int_{v} \mathrm{~d} \boldsymbol{r}_{12}\left(3 \frac{x_{12, i} x_{12, j}}{r_{12}^{2}}-\delta_{i j}\right)\left(\frac{x_{12, k}}{r_{12}^{3}} \frac{r}{\partial x_{12, l}}+\frac{x_{12, l}}{r_{i 2}^{3}} \frac{\partial}{\partial x_{12, h}}\right) .
$$

If averaging is assumed to commute with differentiating and integrating, the average value of $D_{i j}$ equals the average values of $D_{i j}^{(0)}$, because in the term $\left\langle D_{i j}^{(i)}\right\rangle$ the quantity in (4.14),

$$
\left\langle\gamma n_{k} \frac{\partial n_{l}}{\partial x_{12, l}} \delta\left(\boldsymbol{r}_{1}+\boldsymbol{r}_{12}-\boldsymbol{r}_{01}\right)\right\rangle=\frac{1}{V} \int \mathrm{d} \boldsymbol{r}_{1} \gamma n_{k} \frac{\partial n_{l}}{\partial x_{12 . l}} \delta\left(\boldsymbol{r}_{1}+\boldsymbol{r}_{12}-\boldsymbol{r}_{0}\right)=0,
$$

see eq. (38) of Rosenkilde ${ }^{19}$ ), and because in eq. (4.11) the quantity $\left\langle q\left(\boldsymbol{r}_{1}+\boldsymbol{r}_{12}\right) D_{j k}\left(\boldsymbol{r}_{1}+\boldsymbol{r}_{12}\right)\right\rangle$ does not depend on $\boldsymbol{r}_{12}$ since statistical homogeneity is assumed. From this it follows

$$
\left\langle D_{i j}\right\rangle=\left\langle D_{i j}^{(0)}\right\rangle=D_{i j}^{(0)} .
$$

The contribution of the interfacial tension to the rate-of-strain tensor $D^{\text {vanishes }}$ if all the particles are spheres: in that case div $\boldsymbol{n}$ is a constant and that part of the integrand in (4.14) enclosed in parentheses can be written as the curl of $\boldsymbol{r} \times \boldsymbol{r} / \boldsymbol{r}^{3}$, so that Stokes' theorem can be employed for a closed surface. Also in the case of mixtures of fluids $(\gamma=0)$ the tensor $D^{(i)}$ equals zero. Henceforth, only these two special systems are considered, i.e. $\boldsymbol{D}^{(i)}=\mathbf{0}$.

Eq. (4.13) may now be written

$$
D_{i j}=\left\langle D_{i j}\right\rangle+G_{i j k l} q D_{k l} .
$$

The integrand in the integral-operator $G_{i j k l}$ appearing in Cartesian form in $(4.15)$ 
can be written in terms of spherical harmonics $Y_{l m}(\phi, \theta)$ as follows:

$$
\begin{aligned}
G_{i j k l}\left(\boldsymbol{r}_{1}\right)= & \frac{1}{5}\left(\delta_{i k} \delta_{l j}+\delta_{i l} \delta_{k j}-\frac{2}{3} \delta_{i j} \delta_{k l}\right) \frac{1}{4 \pi} \int \mathrm{d} \boldsymbol{r}_{12} \frac{1}{r_{12}^{2}} \frac{\partial}{\partial r_{12}} \\
& +c_{i j k l, \lambda \mu} \frac{1}{4 \pi} \int \mathrm{d} \boldsymbol{r}_{12} \frac{1}{r_{12}^{3}} Y_{i \mu}\left(\hat{\boldsymbol{r}}_{12}\right), \quad \lambda=2,4 ; \mu=-\lambda,-\lambda+1, \ldots, \lambda,
\end{aligned}
$$

where the constants $c_{i j k i, i_{k}}$ connect the Cartesian with the spherical tensors (see Willemse and Caspers $\left.{ }^{10}\right)$ ). These constants $c_{i j k, \lambda \mu}$ are symmetrical in the indices $i$ and $j, k$ and $l$, and also in the pairs of indices $i j$ and $k l$ (cf. eq. (93) of Kröner and $\left.\mathrm{Koch}^{20}\right)$ ).

The values of the complex numbers $c_{i j k, \lambda \mu}$ are immaterial within the scope of this paper, but it is important to note the following properties, which can be derived from eq. (4.20):

$$
c_{i j i j, \lambda \mu}=c_{i i j j, \lambda \mu}=c_{i j j, \lambda \mu}=0 .
$$

The first integral is a total derivative with respect to the radius $r$, from which it follows that eq. (4.19) can be written as

with

$$
D_{i j}=\left\langle D_{i j}\right\rangle+\frac{2}{5}\left\langle q D_{i j}\right\rangle-\frac{2}{5} q D_{i j}+Y_{i j k l} q D_{k l},
$$

$$
Y_{i j k l}\left(\boldsymbol{r}_{1}\right)=c_{i j k l, \lambda \mu} \frac{1}{4 \pi} \int \mathrm{d} \boldsymbol{r}_{12} \frac{1}{\boldsymbol{r}_{12}^{3}} Y_{i, \mu}\left(\hat{\boldsymbol{r}}_{12}\right) .
$$

Keeping in mind that $(4.21)$ represents an iteration series, one can also represent this equation by its sum:

$$
D_{i j}=\left(\frac{\langle D\rangle+\frac{2}{5}\langle q D\rangle}{1+\frac{2}{5} q-Y q}\right)_{i j}
$$

Expansion of this series in the first few terms should lead to

$$
D_{i j}=\frac{1}{1+\frac{2}{5} q}\left\{\left(1+Y_{i j k l} \frac{q}{1+\frac{2}{5} q}+Y_{i j k l}^{2} \frac{q^{2}}{\left(1+\frac{2}{5} q\right)^{2}}+\cdots\right)\left(\left\langle D_{k l}\right\rangle+\frac{2}{5}\left\langle q D_{k l}\right\rangle\right)\right\},
$$

where the third term written out in full reads

$$
Y_{i j k l}^{2}=c_{i m n n, \dot{\mu} \mu} c_{m n k l, \lambda^{\prime} \mu^{\prime}} \frac{1}{(4 \pi)^{2}} \int \mathrm{d} \boldsymbol{r}_{12} \frac{1}{r_{12}^{3}} Y_{\lambda \mu}\left(\hat{r}_{12}\right) \int \mathrm{d} \boldsymbol{r}_{23} \frac{1}{r_{23}^{3}} Y_{\dot{\lambda}^{\prime} \mu^{\prime}}\left(\hat{\boldsymbol{r}}_{23}\right) .
$$

\section{Calculation of the effective viscosity and its bounds}

The definition of the effective viscosity $\eta^{*}$, given by eq. (3.1) and in another form by eq. (3.11), results in combination with (4.5) in the task to calculate

$$
\eta^{*}\left\langle D_{i j}\right\rangle=\eta_{0}\left\langle(1+q) D_{i j}\right\rangle+\frac{1}{2} c\left\langle D_{i j}\right\rangle
$$


where $c$ is given in eq. (3.5),

$$
\left\langle\gamma\left(\frac{1}{3} \delta_{i j}-n_{i} n_{j}\right) \delta\left(\boldsymbol{r}-\boldsymbol{r}_{i}\right)\right\rangle=c\left\langle D_{i i}\right\rangle .
$$

In the last equation the constant $c$ expresses the relation between the averaged rate-of-strain exposed on the dispersion and the resulting form of the fluid particles. Starting from a dead fluid with spherical particles, the imposed fluid flow in the neighbourhood of the particle determines the deformation of the spherical particle and the deformed sphere in its turn determines the fluid flow. In other words, one has to solve an equilibrium problem, such that the equation $\left[\sigma_{i j}\right] n_{j}=\gamma n_{i}$ div $n$ holds. This constitutes a difficult problem with boundary conditions on boundaries that depend themselves on the solution. It appears that this problem cannot be solved with the method presented here, but that one has to solve a deterministic problem in order to determine the value of the constant $c$. By not taking into account this term any longer, it is to be expected that the results in the remaining of this paper can only be valid for dispersions of spherical particles, which may be realized by letting $\eta_{2}$ go to infinity at constant $\gamma$, so that eq. (5.2) gives no contribution to eq. (5.1) and also in the case of $\gamma$ going to zero giving rise to all kinds of deformations of the particles, such that $\left\langle\left(\frac{1}{3} \delta_{i j}-n_{i} n_{j}\right) \delta\left(\boldsymbol{r}-\boldsymbol{r}_{0}\right)\right\rangle$ goes to zero. In these two cases eq. (5.1) simplifies to

$$
\eta^{*}\left\langle D_{i j}\right\rangle=\eta_{0}\left\langle(1+q) D_{i j}\right\rangle=\eta_{0}\left\langle D_{i j}\right\rangle+\eta_{0}\left\langle q D_{i i}\right\rangle .
$$

From eq. (4.23) it follows

$$
\left\langle q D_{i j}\right\rangle=\left\langle q\left(\frac{\langle D\rangle+\frac{2}{5}\langle q D\rangle}{1+\frac{2}{5} q-Y q}\right)_{i i}\right\rangle,
$$

and taking the term $1+\frac{2}{5} q$ out of the parentheses, one gets

$$
\begin{aligned}
\left\langle q D_{i j}\right\rangle & =\left\langle\frac{q}{1+\frac{2}{5} q}\left(\frac{\langle D\rangle+\frac{2}{5}\langle q D\rangle}{1-Y \frac{q}{1+\frac{2}{5} q}}\right)\right\rangle \\
& =\left\langle\frac{\tilde{\eta}}{1-Y \tilde{\eta}}\right\rangle_{i j k l}\left\langle D_{k l}\right\rangle+\frac{2}{\overline{5}}\left\langle\frac{\tilde{\eta}}{1-Y \tilde{\eta}}\right\rangle_{i j k l}\left\langle q D_{k l}\right\rangle,
\end{aligned}
$$

with

$$
\tilde{\eta} \equiv \frac{q}{1+\frac{2}{5} q} .
$$

Eq. (4.5) being used, the renormalized parameter $\tilde{\eta}$ can also be written as

$$
\tilde{\eta}=5 \frac{\eta-\eta_{0}}{2 \eta+3 \eta_{0}}
$$

In order to obtain an explicit expression for $\left\langle q D_{i j}\right\rangle$ the second term in the 
right-hand side of eq. (5.5) is taken to the left-hand side of this equation, after which the resulting equation is multiplied by the inverse of the fourth-rank tensor

$$
\left(1-\frac{2}{5}\left\langle\frac{\tilde{\eta}}{1-Y \tilde{\eta}}\right\rangle\right)_{i j k l} .
$$

The solution of (5.5) is then given by

$$
\left\langle q D_{i j}\right\rangle=\frac{\left\langle\frac{\tilde{\eta}}{1-Y \tilde{\eta}}\right\rangle_{k l m n}}{\delta_{i k} \delta_{j l}-\frac{2}{5}\left\langle\frac{\tilde{\eta}}{1-Y \tilde{\eta}}\right\rangle_{i j k l}}\left\langle D_{m n}\right\rangle,
$$

where in the series expansion of the formal geometrical progressions the adequate contraction of the tensors must be performed.

Equation (5.3) can now be written as

$$
\begin{aligned}
\eta^{*}\left\langle D_{i j}\right\rangle & =\eta_{0}\left[\delta_{i m} \delta_{j n}+\frac{\left\langle\frac{\tilde{\eta}}{1-Y \tilde{\eta}}\right\rangle_{k i m n}}{\delta_{i k} \delta_{j l}-\frac{2}{5}\left\langle\frac{\tilde{\eta}}{1-Y \tilde{\eta}}\right\rangle_{i j k l}}\right]\left\langle D_{m n}\right\rangle \\
& =\eta_{0}\left[\frac{\delta_{k m} \delta_{l n}+\frac{3}{5}\left\langle\frac{\tilde{\eta}}{1-Y \tilde{\eta}}\right\rangle_{k i m n}}{\delta_{i k} \delta_{j l}-\frac{2}{5}\left\langle\frac{\tilde{\eta}}{1-Y \tilde{\eta}}\right\rangle_{i j k l}}\right]\left\langle D_{m n}\right\rangle .
\end{aligned}
$$

Since the dispersion behaves statistically isotropic, the expression in square brackets in (5.8) should be an isotropic tensor. For this reason the effective viscosity can be represented by the following scalar:

$$
\eta^{*}=\eta_{0} \frac{1+(3 / 5) \sum_{n=1}^{\infty} T^{(n)}}{1-(2 / 5) \sum_{n=1}^{\infty} T^{(n)}},
$$

with

$$
T^{(n)}=\frac{1}{10} T_{i j i j}^{(n)}, \quad n=1,2, \ldots
$$

The first three terms of the $T$-series read

$$
\begin{aligned}
& T^{(1)}=\langle\tilde{\eta}\rangle=\left\langle 5 \frac{\eta-\eta_{0}}{2 \eta+3 \eta_{0}}\right\rangle, \\
& T_{i j k l}^{(2)}=c_{i j k l, i \mu \mu} \frac{1}{4 \pi} \int \mathrm{d} \boldsymbol{r}_{12} \frac{1}{r_{12}^{3}} Y_{i \mu}\left(\hat{r}_{12}\right)\left\langle\tilde{\eta}\left(\boldsymbol{r}_{1}\right) \tilde{\eta}\left(\boldsymbol{r}_{1}+\boldsymbol{r}_{12}\right)\right\rangle,
\end{aligned}
$$




$$
\begin{aligned}
& T_{i j k l}^{(3)}=c_{i j m n, \lambda \mu} c_{m m k l, i, \mu} \\
& \times \frac{1}{(4 \pi)^{2}} \int \mathrm{d} \boldsymbol{r}_{12} \int \mathrm{d} \boldsymbol{r}_{23} \frac{1}{r_{12}^{3}} r_{23}^{3} Y_{\lambda, \mu}\left(\hat{\boldsymbol{r}}_{12}\right) Y_{, \mu,}\left(\hat{\boldsymbol{r}}_{23}\right) \\
& \times\left\langle\tilde{\eta}\left(\boldsymbol{r}_{1}\right) \bar{\eta}\left(\boldsymbol{r}_{1}+\boldsymbol{r}_{12}\right) \tilde{\eta}\left(\boldsymbol{r}_{1}+\boldsymbol{r}_{12}+\boldsymbol{r}_{23}\right)\right\rangle .
\end{aligned}
$$

Note that the first term $T^{\prime 11}$ is texture-independent, i.e. it does not depend on the form, size distribution, orientation, orientation distribution, spatial distribution. and other statistical information regarding the geometry of the fluid particles, and that the higher-order terms $T^{(n)}, n \geqslant 2$, express in increasing detail the influence of the textural properties of the dispersion on the effective viscosity because $T^{(n)}$, $n \geqslant 2$. represent angular integrals over $n$-point correlation functions (Beran ${ }^{7}$ )).

The value of $\eta_{0}$ is still undetermined and can be chosen at will. The value of $\eta_{0}$ following from the obvious condition $\langle\tilde{\eta}\rangle=0$ is the value corresponding with the effective viscosity $\eta^{*}$ in the effective-medium theory $\left(\right.$ Hori $\left.^{21}\right)$ ). This condition leads to the quadratic equation

$$
\eta_{11}^{2}-\frac{1}{3} \eta_{0}\left(\eta_{1}(3-5 \phi)-\eta_{2}(2-5 \phi)\right)-\frac{2}{3} \eta_{1} \eta_{2}=0 .
$$

The one positive root of this equation is given by the familiar solution of quadratic equations, but in two limiting cases a simple expression is obtained,

$$
\begin{aligned}
& \eta_{2} \rightarrow \infty: \quad \eta_{0}=\frac{\eta_{1}}{1-\frac{5}{2} \phi} . \\
& \eta_{2} \rightarrow 0: \quad \eta_{0}=\eta_{1}\left(1-\frac{3}{3} .\right.
\end{aligned}
$$

The viscosity in eq. (5.15), which should be considered as the texture-independent part of the effective viscosity, coincides with the effective viscosity derived by Lundgren ${ }^{6}$ ).

In general the solution of (5.14) can be expressed in first order of $\phi$.

$$
\eta_{0}=\eta_{1}\left(1+\frac{-5 \eta_{1}+5 \eta_{2}}{3 \eta_{1}+2 \eta_{2}} \phi\right)
$$

This result can be regarded as the texture-independent viscosity at low concentrations and may be compared with the Einstein-Taylor formula

$$
\eta^{*}=\eta_{1}\left(1+\frac{2 \eta_{1}+5 \eta_{2}}{2 \eta_{1}+2 \eta_{2}} \phi\right)
$$

Only in the limiting case of rigid spheres $\eta_{2} \rightarrow \infty$ these two expressions give the same result, which is not at all surprising since the effect of the interfacial tension is completely ignored in all other cases in (5.17).

The freedom of choice of the value of $\eta_{0}$ enables one to establish the so-called 
Hashin-Shtrikman bounds (Hashin and Shtrikman ${ }^{22}$ )):

$$
A \leqslant \eta^{*} \leqslant B,
$$

in which

$$
A=\eta_{0} \frac{1+\frac{3}{5} T^{(1)}}{1-\frac{2}{5} T^{(1)}}, \quad \text { with } \eta_{0} \leqslant \min \left\{\eta_{1}, \eta_{2}\right\}
$$

and

$$
B=\eta_{0} \frac{1+\frac{3}{5} T^{(1)}}{1-\frac{2}{5} T^{(1)}}, \quad \text { with } \eta_{0} \geqslant \max \left\{\eta_{1}, \eta_{2}\right\} .
$$

By taking $\eta_{0}=\min \left\{\eta_{1}, \eta_{2}\right\}$ and $\eta_{0}=\max \left\{\eta_{1}, \eta_{2}\right\}$ these bounds are the best possible bounds on the basis of the one-point correlation function alone, i.e. in terms of $\phi, \eta_{1}$ and $\eta_{2}$ alone. If it is assumed that $\eta_{1} \leqslant \eta_{2}$, these best bounds become

$$
\eta_{1} \frac{3 \eta_{1}+2 \eta_{2}+3\left(\eta_{2}-\eta_{1}\right) \phi}{3 \eta_{1}+2 \eta_{2}-2\left(\eta_{2}-\eta_{1}\right) \phi} \leqslant \eta^{*} \leqslant \eta_{2} \frac{5 \eta_{1}+3\left(\eta_{2}-\eta_{1}\right) \phi}{5 \eta_{2}-2\left(\eta_{2}-\eta_{1}\right) \phi}
$$

if $\eta_{1}>\eta_{2}$, the inequality signs must be reversed. For $\eta_{0} \rightarrow 0$ respectively $\eta_{0} \rightarrow \infty$ the bounds given in eq. (3.18) are reproduced,

$$
\left\langle\eta^{-1}\right\rangle^{-1} \leqslant \eta^{*} \leqslant\langle\eta\rangle \text {. }
$$

For the two limiting cases mentioned above, $\eta_{2} \rightarrow \infty$ and $\eta_{2} \rightarrow 0$, a lower bound respectively an upper bound can be deduced

$$
\begin{aligned}
& \eta_{2} \rightarrow \infty: \quad \eta^{*} \geqslant \eta_{1} \frac{2+3 \phi}{2(1-\phi)}=\eta_{1}\left(1+\frac{5}{2} \phi+\frac{5}{2} \phi^{2}+\cdots\right), \\
& \eta_{2} \rightarrow 0: \quad \eta^{*} \leqslant \eta_{1} \frac{1-\phi}{1+\frac{2}{3} \phi}=\eta_{1}\left(1-\frac{5}{3} \phi+\frac{10}{9} \phi^{2}+\cdots\right) .
\end{aligned}
$$

From eq. (5.22) it follows that the so-called Huggins coefficient $K_{\mathrm{H}}$, determining the second-order term of $\eta^{*}$ in the case of rigid particles,

$$
\eta^{*}=\eta_{1}\left(1+\frac{5}{2} \phi+K_{\mathrm{H}}\left(\frac{5}{2} \phi\right)^{2}+\cdots\right),
$$

possesses a lower bound:

$$
K_{\mathrm{H}} \geqslant 0.4 \text {. }
$$

\section{Discussion}

The results derived by the presented statistical continuum approach are only valid in cases that $\langle\boldsymbol{\Gamma}\rangle$ and $\boldsymbol{D}^{(\gamma)}$ equal zero. This imperfection is difficult to 
eliminate by reasons explained in section 5 . Both requirements are satisfied for rigid spheres and for mixtures of Newtonian fluids with vanishing interfacial tension.

To get some insight in the influence of $\langle\boldsymbol{\Gamma}\rangle$ and $\boldsymbol{D}^{(i)}$ an analysis of the well-known deterministic calculation of the effective viscosity of an emulsion of two Newtonian fluids in the first order of volume fraction of the (almost) spherical particles is instructive. A convenient starting point is the general expression of the stress tensor for an emulsion given by Batchelor ${ }^{18}$ ),

$$
\langle\Sigma\rangle=(\text { pressure term })+2 \eta_{1}\langle\boldsymbol{D}\rangle+\lim _{\boldsymbol{V} \rightarrow x} \frac{1}{V} \int_{S_{p}}\left(\boldsymbol{\sigma}_{1} \cdot \boldsymbol{n} \boldsymbol{r}-\eta_{1}(\boldsymbol{u} \boldsymbol{n}+\boldsymbol{n u})\right) \mathrm{d} S, \text { (6.1) }
$$

where $\partial V_{\mathrm{p}}$ is the surface of the particles within $V$ and $\sigma_{1}$ is the stress tensor of the Newtonian continuous phase. With the help of eq. (2.12) and Rosenkilde's ${ }^{14}$ ) equations (8) and (15) it follows that

$$
\int_{i V_{\mathrm{p}}}\left(\boldsymbol{\sigma}_{1}-\boldsymbol{\sigma}_{2}\right) \cdot \boldsymbol{n} \boldsymbol{r} \mathrm{d} S=\int_{i_{\mathrm{p}}} \gamma \boldsymbol{n} \boldsymbol{r} \operatorname{div} \boldsymbol{n} \mathrm{d} S=\int_{V_{\mathrm{p}}} \gamma \boldsymbol{U}_{\mathrm{II}} \mathrm{d} S .
$$

The stress tensor $\boldsymbol{\sigma}_{2}$ belongs to the Newtonian dispersed phase given by

$$
\sigma_{2}=-p \boldsymbol{T}+2 \eta_{2} D
$$

Since $\boldsymbol{\nabla} \cdot \boldsymbol{\sigma}_{2}=0$ within the particles, it follows, with Gauss' theorem,

$$
\int_{r V_{\mathrm{p}}} \boldsymbol{\sigma}_{2} \cdot \boldsymbol{n} \boldsymbol{r} \mathrm{d} S=\int_{V_{\mathrm{p}}} \boldsymbol{\sigma}_{2} \mathrm{~d} V=\eta_{2} \int_{V_{\mathrm{p}}}(\boldsymbol{u n}+\boldsymbol{n u}) \mathrm{d} S+(\text { pressure term }) .
$$

Thus eq. (6.1) becomes

$$
\begin{aligned}
\langle\Sigma\rangle= & \text { (pressure term })+2 \eta_{1}\langle\boldsymbol{D}\rangle \\
& +\lim _{V^{\prime} \rightarrow x} \frac{1}{V}\left(\eta_{2}-\eta_{1}\right) \int_{i V_{\mathrm{p}}}(\boldsymbol{u} \boldsymbol{n}+\boldsymbol{n u}) \mathrm{d} S+\lim _{\boldsymbol{V} \rightarrow x} \frac{1}{V} \int_{V_{\mathrm{p}}} \gamma \boldsymbol{U}_{\mathrm{II}} \mathrm{d} S .
\end{aligned}
$$

Apparently

$$
2 \eta_{1}\langle\boldsymbol{D}\rangle+\lim _{V \rightarrow x} \frac{1}{V}\left(\eta_{2}-\eta_{1}\right) \int_{i V_{p}}(\boldsymbol{u n}+\boldsymbol{n u}) \mathrm{d} S
$$

and the deviatoric part of

$$
\lim _{V^{\prime} \rightarrow x} \frac{1}{V} \int_{i V_{p}} \gamma \boldsymbol{U}_{\mathrm{II}} \mathrm{d} S
$$


correspond to

$$
2 \eta_{0}\langle(1+q) \boldsymbol{D}\rangle \text { and }\left\langle\gamma\left(\frac{1}{3} \boldsymbol{1}-\boldsymbol{n n}\right) \delta\left(\boldsymbol{r}-\boldsymbol{r}_{0}\right)\right\rangle,
$$

respectively, of the present paper.

Two conclusions can be drawn at first sight. For $\gamma=0$ the effective viscosity of the emulsion is $\eta_{1}$ plus a quantity proportional to $\eta_{2}-\eta_{1}$, which is confirmed by expression (5.17). Secondly, for arbitrary $\gamma$ the form of the particles will influence the flow field and in general $\int_{\partial V_{\mathrm{p}}}(\boldsymbol{u n}+\boldsymbol{n} \boldsymbol{u}) \mathrm{d} S$, thus making $\eta^{*}$ dependent on $\gamma$. However, apart from the contribution through $\langle\boldsymbol{\Gamma}\rangle$, it is not clear whether $\gamma$ contributes to $\eta^{*}$ by means of the texture-dependent part of it or directly by $D^{(\gamma)}$ or by both.

The papers of Schowalter et al. ${ }^{23}$ ) and Taylor ${ }^{24}$ ) enhance these conclusions for emulsions in a pure straining motion. Schowalter et al..$^{23}$ ) calculated the viscosity of almost spherical particles $(\gamma \rightarrow \infty)$ of Newtonian fluid with viscosity $\eta_{2}$ dispersed in a Newtonian fluid with viscosity $\eta_{1}$ in the first order of volume fraction $\phi$, using expression (6.1). Their result (see appendix D) is

$$
\lim _{V \rightarrow \infty} \frac{1}{V}\left(\eta_{2}-\eta_{1}\right) \int_{\partial V_{\mathrm{p}}}(\boldsymbol{u n}+\boldsymbol{n u}) \mathrm{d} S=\frac{6}{5} \frac{\eta_{2}-\eta_{1}}{\eta_{1}+\eta_{2}} \eta_{1} \phi\langle\boldsymbol{D}\rangle
$$

and

$$
\lim _{V \rightarrow \infty} \frac{1}{V} \int_{\partial V_{\mathrm{p}}} \gamma\left(\frac{1}{3} \boldsymbol{1}-\boldsymbol{n n}\right) \mathrm{d} S=\frac{16 \eta_{1}+19 \eta_{2}}{5\left(\eta_{1}+\eta_{2}\right)} \eta_{1} \phi\langle\boldsymbol{D}\rangle .
$$

If (6.6) and (6.7) are added, the Einstein-Taylor contribution to the effective viscosity can be deduced. The formalism in the paper of Schowalter et al. ${ }^{23}$ ) and the calculations in Taylor's paper ${ }^{24}$ ) can be used to deduce the contribution of both terms for the case of an emulsion of Newtonian fluids with viscosities $\eta_{1}$ and $\eta_{2}$ and interfacial tension $\gamma=0$ during the first moment when spheres of Newtonian fluid with viscosity $\eta_{2}$ are placed in a pure straining motion. It appears that for this case

$$
\lim _{V \rightarrow \infty} \frac{1}{V}\left(\eta_{2}-\eta_{1}\right) \int_{\partial V_{p}}(\boldsymbol{u n}+\boldsymbol{n u}) \mathrm{d} S=\frac{10\left(\eta_{2}-\eta_{1}\right)}{3 \eta_{1}+2 \eta_{2}} \eta_{1} \phi\langle\boldsymbol{D}\rangle
$$

and

$$
\lim _{V \rightarrow \infty} \frac{1}{V} \int_{\partial V_{\mathrm{p}}} \gamma\left(\frac{1}{3} \boldsymbol{1}-\boldsymbol{n n}\right) \mathrm{d} S=0
$$


Eq. (6.8) results in a viscosity change

$$
\eta_{1} \frac{5\left(\eta_{2}-\eta_{1}\right)}{3 \eta_{1}+2 \eta_{2}} \phi
$$

due to the dispersed phase, as is also found in expression (5.17). As expected, (6.6) and (6.8) are proportional to $\eta_{2}-\eta_{1}$. Since in both cases the form of the particles is almost spherical, the difference between (6.6) and (6.8) is due to the influence of the tensor $\boldsymbol{D}^{(\text {) })}$.

Experimentally (5.14) can be checked if the tractions on either side of the interface are much larger than their difference (see eq. (2.12)). This is the case for the dynamic viscosity if the frequency is large enough. For the special case $\phi \rightarrow 0$ Oldroyd $^{25}$ ) derived that the high-frequency real part of the dynamic viscosity equals the viscosity as given by (5.17). The feasibility of pertinent measurements has been demonstrated by Oosterbroek et al. ${ }^{26}$ ).

As a method the presented statistical continuum approach can be compared with work done by Nagatani") and Bedeaux et al. ${ }^{12}$ ) for suspensions of spheres. They also solved the flow equations formally with generalized Oseen operators. but they chose the way via Fourier transformation of the flow equation. Nagatani's result,

$$
\eta^{*}=\eta_{1}\left[1+\frac{5\left(\eta_{1}-\eta_{2}\right)}{3 \eta_{1}+2 \eta_{2}} \phi\right]^{\prime}
$$

deduced for $\gamma=0$, may be compared with (5.14). In the first order of $\phi$ expressions (6.10) and (5.14) are the same. Also both expressions of $\eta^{*}$ satisfy the condition that for $\eta_{1}=\eta_{2}$ the effective viscosity equals $\eta_{1}$. For $\eta_{1} \neq \eta_{2}$ the expressions differ in higher order of $\phi$. The cause of this difference is not clear. Note that for $\eta_{2} \rightarrow \propto$ (rigid particles) the difference disappears.

The results of Bedeaux et al. ${ }^{12}$ ) are less easy to compare because they presented the effective viscosity as an operator. After some approximation it degenerates to a scalar, for which they presented two expressions. First they calculated the viscosity in the absence of number density correlations,

$$
\eta^{*}=\eta_{1}\left(1+\frac{5}{2} \phi+\frac{5}{2} \phi^{2}+\cdots\right)
$$

and further $\eta^{*}$ was calculated up to the second order in the density of the spheres,

$$
\eta^{*}=\eta_{1}\left(1+\frac{5}{2} \phi+4.8 \phi^{2}\right)
$$

Apparently, both results are less accurate than the effective viscosity of Nagatani and the effective medium viscosity of the present paper, for which the Batchelor-Green expression (1.3) for $\eta^{*}$ is taken as the most reliable reference. Eq. (6.11) is even extremely low since it is identical with the lower bound in inequality (5.22). 
Another result that is noteworthy but methodically derived in a different way was given by Lundgren ${ }^{6}$ ). He proposed an ad hoc closure of an hierarchy of flow equations (see section 1). Physically his approach implies that statistically each rigid sphere moves in a medium with effective viscosity

$$
\eta^{*}=\eta_{1}\left(1-\frac{5}{2} \phi\right)^{-1}
$$

but it is interpreted as an approximation of the effective viscosity of the dispersion. Expression (6.13) is identical with the effective medium viscosity given in (5.15).

The bounds given in the inequalities (5.20) are identical with the bounds obtained by $\mathrm{Hashin}^{8}$ ), using a variational method. Again their validity is restricted to mixtures of Newtonian fluids with $\gamma=0$ and to rigid spheres $\left(\eta_{2} \rightarrow \infty\right)$. The lower bound, (5.22), may also be compared with the corresponding bound given by Keller et al. $^{13}$ ), which they deduced using extremum principles for a cellular system. The lower bound, given in (5.22), appears to be an improvement upon theirs, for it is higher. The other bounds, given in (5.21), which were also found by Hashin $^{8}$ ) and Batchelor ${ }^{14}$ ), are worse than those in (5.20). That this should be the case was demonstrated by Hashin $^{8}$ ).

The main merit of the statistical continuum approach given in the present paper appears to be the deduction of an effective medium viscosity and viscosity bounds from one point of view and a contribution to the interpretation of the results, also found by $\operatorname{Hashin}^{8}$ ), Lundgren ${ }^{6}$ ), Batchelor ${ }^{14}$ ), and Nagatani ${ }^{11}$ ), having been derived after omission of $\langle\boldsymbol{\Gamma}\rangle$ and $\boldsymbol{D}^{(\eta)}$.

\section{Appendix A}

\section{Conservation of momentum}

In the derivation of eq. (2.11) two starting-points are used, namely Newton's law in the steady-state approximation and the constitutive equation for the dispersion.

Newton's law:

$$
\int_{S} \Sigma \cdot N \mathrm{~d} S=0,
$$

(see fig. 1).

Constitutive equation:

$$
\boldsymbol{\Sigma}=\sigma+\gamma \boldsymbol{U}_{\mathrm{II}} \delta\left(\boldsymbol{r}-\boldsymbol{r}_{0}\right)
$$

Here the tensor $\boldsymbol{U}_{\mathrm{II}}$ denotes the second-rank unity tensor, having the components $\left(\delta_{i j}-n_{i} n_{j}\right)$ in a Cartesian frame of reference. 


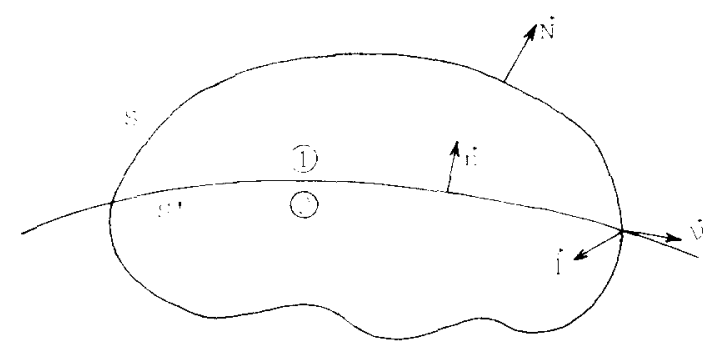

Fig. 1. Intersection of an arbitrary volume $V$ with boundary $S$ containing part of the interface $S^{\prime}$ of a fluid particle and the ambient fluid.

Combination of (A.1) and (A.2) gives

$$
\begin{aligned}
0 & =\int_{S} \boldsymbol{\Sigma} \cdot \boldsymbol{N} \mathrm{d} S=\int_{S} \boldsymbol{\sigma} \cdot \boldsymbol{N} \mathrm{d} S+\int_{S} \gamma \boldsymbol{U}_{\mathrm{II}} \cdot \boldsymbol{N} \delta\left(\boldsymbol{r}-\boldsymbol{r}_{0}\right) \mathrm{d} S \\
& =\int_{S} \sigma_{i j} N_{j} \mathrm{~d} S+\oint_{C} \gamma\left(\delta_{i j}-n_{i} n_{j}\right) N_{j} \mathrm{~d} s .
\end{aligned}
$$

The path-integral in (A.3) is performed over a closed curve $C$, being the intersection of the surfaces $S$ and $S^{\prime}$. The projection of the unit normal vector $N$ of the surface $S$ on the surface $S^{\prime}$ results in a unit vector $v$ tangential to $S^{\prime}$ and perpendicular to $C$. This implies that $v=N$, so that the otherwise arbitrary surface $S$ should intersect the interface $S^{\prime}$ perpendicularly. The subtle reason for this condition is to provide antagonistic interior and exterior points of the fluid particle on either side of the interface $S^{\prime}$; this choice is a matter of convenience. The triplet $\boldsymbol{n}, \boldsymbol{l}$ and $\boldsymbol{v}$ constitute a right-handed coordinate system on the curve $C, \boldsymbol{l}$ being the unit tangent vector of the curve $C$.

Equation (A.3) then becomes

$$
0=\int_{S} \Sigma \cdot N \mathrm{~d} S=\int_{S} \sigma \cdot N \mathrm{~d} S+\oint_{\zeta} \gamma v \mathrm{~d} s,
$$

and on applying Gauss' theorem,

$$
\begin{aligned}
0=\int_{S} \Sigma \cdot N \mathrm{~d} S= & \int_{V_{1}} \frac{\partial}{\partial x_{j}} \sigma_{i j} \mathrm{~d} V+\int_{S_{1}} \sigma_{i j} n_{j} \mathrm{~d} S \\
& +\int_{V_{2}} \frac{\partial}{\partial x_{j}} \sigma_{i j} \mathrm{~d} V-\int_{S_{2}} \sigma_{i j} n_{j} \mathrm{~d} S+\oint_{C} \gamma v_{i} \mathrm{~d} s .
\end{aligned}
$$


Analogously to the description of $\eta$ as given in (2.7) the stress tensor $\sigma$ can be given by

$$
\sigma_{i j}=\sigma_{1, i j}(1-\xi)+\sigma_{2, i j} \xi
$$

Differentiation with respect to the coordinate $r$ gives

$$
\frac{\partial}{\partial x_{j}} \sigma_{i j}=(1-\zeta) \frac{\partial}{\partial x_{j}} \sigma_{1, i j}+\xi \frac{\partial}{\partial x_{j}} \sigma_{2, i j}+\left(\sigma_{1, i j}-\sigma_{2, i j}\right) n_{j} \delta\left(\boldsymbol{r}-\boldsymbol{r}_{0}\right) .
$$

Keeping in mind this way of dealing with discontinuities, one may write (A.5) as

$$
0=\int_{S} \Sigma \cdot N \mathrm{~d} S=\int_{V} \frac{\partial}{\partial x_{j}} \sigma_{i j} \mathrm{~d} V+\oint_{C} \gamma v_{i} \mathrm{~d} s .
$$

For the evaluation of the contour-integral Stokes' theorem is applied on a tensor $\boldsymbol{A}=\gamma \boldsymbol{n} \otimes$ or indexical $A_{k l}=\gamma \epsilon_{k p l} n_{p}$, thus following Rosenkilde's proof ${ }^{19}$ ) of eq. (9) but now for an open surface:

$$
\begin{aligned}
\oint_{C} \boldsymbol{A} \cdot \boldsymbol{l} \mathrm{d} s & =\int_{S^{\prime}}(\boldsymbol{\nabla} \times \boldsymbol{A}) \cdot \boldsymbol{n} \mathrm{d} S \\
& =\int_{S^{\prime}} \epsilon_{i j k}\left(\frac{\partial}{\partial x_{j}} A_{k l}\right) n_{i} \mathrm{~d} S \\
& =\int_{S^{\prime}} \epsilon_{i j k}\left(\frac{\partial}{\partial x_{j}} \gamma \epsilon_{k p l} n_{p}\right) n_{i} \mathrm{~d} S \\
& =\int_{S^{\prime}}\left(-\gamma n_{l} \frac{\partial n_{j}}{\partial x_{j}}+\left(\delta_{l j}-n_{l} n_{j}\right) \frac{\partial \gamma}{\partial x_{j}}\right) \mathrm{d} S
\end{aligned}
$$

Since the triplet $\boldsymbol{n}, \boldsymbol{l}$, and $\boldsymbol{v}$ constitute a right-handed system, it holds $\boldsymbol{n} \times \boldsymbol{l}=\boldsymbol{v}$ and therefore $\boldsymbol{A} \cdot \boldsymbol{l}=\gamma \boldsymbol{v}$. In (A.9) use is made of the identity

$$
n_{i} \frac{\partial}{\partial x_{j}} n_{i}=\frac{1}{2} \frac{\partial}{\partial x_{j}} n_{i} n_{i}=\frac{1}{2} \frac{\partial}{\partial x_{j}} 1=0 .
$$

Returning to eq. (A.8):

$$
\begin{aligned}
0 & =\int_{S} \Sigma \cdot N \mathrm{~d} S=\int_{V} \frac{\partial}{\partial x_{j}} \sigma_{i j} \mathrm{~d} V+\int_{S^{\prime}}\left(-\gamma n_{i} \frac{\partial n_{j}}{\partial x_{j}}+\left(\delta_{i j}-n_{i} n_{j}\right) \frac{\partial \gamma}{\partial x_{j}}\right) \mathrm{d} S \\
& =\int_{V}\left\{\frac{\partial}{\partial x_{j}} \sigma_{i j}+\left(-\gamma n_{i} \frac{\partial n_{j}}{\partial x_{j}}+\left(\delta_{i j}-n_{i} n_{j}\right) \frac{\partial \gamma}{\partial x_{j}}\right) \delta\left(\boldsymbol{r}-\boldsymbol{r}_{0}\right)\right\} \mathrm{d} V .
\end{aligned}
$$


Since the volume $V$ was taken arbitrarily, it holds

$$
\boldsymbol{\nabla} \cdot \boldsymbol{\sigma}+\nabla_{\mathrm{ll}} \cdot \boldsymbol{\Gamma}=0
$$

where the symbol $\boldsymbol{V}_{\mathrm{II}}=\boldsymbol{U}_{\mathrm{II}} \cdot \boldsymbol{V}$ (see Eliassen $\left.{ }^{27}\right)$ ).

Returning to eq. (A.2), one finally arrives at

$$
\nabla \cdot \Sigma=0 \text {. }
$$

In the limit of $S$ approaching and still enveloping $S^{\prime}$, the vectors $\boldsymbol{n}$ and $N$ will almost everywhere coincide (possibly apart from their sign), so that at the interface $S^{\prime}$ it holds

$$
\left[\sigma_{i j}\right] n_{j}=\gamma n_{i} \frac{\partial n_{j}}{\partial x_{j}}-\frac{\partial \gamma}{\partial x_{j}}\left(\delta_{i j}-n_{i} n_{j}\right)
$$

where the square bracket $\left[\sigma_{i j}\right]$ means the difference between the outside value and the inside value of $\sigma_{i j}$; this is a direct consequence of the inversion of the direction of the vector $N$ at both sides of the interface $S^{\prime}$.

\section{Appendix B}

The average of the stress tensor 2

The average of the stress tensor $\Sigma$ is computed as follows:

$$
\left\langle\Sigma_{i j}\right\rangle=\frac{1}{V} \int_{i} \Sigma_{i j} \mathrm{~d} V=\frac{1}{V} \int_{i}\left\{\sigma_{i j}+\gamma\left(\dot{\delta}_{i j}-n_{i} n_{j}\right) \dot{\delta}\left(\boldsymbol{r}-\boldsymbol{r}_{0}\right)\right\} \mathrm{d} V .
$$

When use is made of eq. (14) in the paper of Rosenkilde ${ }^{19}$ ) for variable $;$,

$$
\int_{i} \gamma\left(\boldsymbol{\delta}_{i j}-n_{i} n_{j}\right) \delta\left(\boldsymbol{r}-\boldsymbol{r}_{0}\right) d V=\int_{i} x_{j}\left(\gamma n_{i} \frac{\partial n_{k}}{\partial x_{k}}-\frac{\partial \gamma}{\partial x_{k}}\left(\delta_{i k}-n_{i} n_{k}\right)\right) \delta\left(\boldsymbol{r}-\boldsymbol{r}_{1}\right) \mathrm{d} V .
$$

eq. (B.1) becomes, with the help of (A.10)

$$
\left\langle\Sigma_{i j}\right\rangle=\left\langle\sigma_{i j}\right\rangle+\left\langle x_{j} \frac{\partial}{\partial x_{k}} \sigma_{i k}\right\rangle=\left\langle\frac{\partial}{\partial x_{k}} \sigma_{i k} x_{i}\right\rangle .
$$

\section{Appendix C}

\section{Hashin-Shtrikman bounds}

The $T$ series is the average of a Neumann series times $\tilde{\eta}$.

$$
\left.\sum_{n=1}^{j} T_{i j k l}^{(n)}=\left\langle\tilde{\eta}\left(\delta_{i k} \delta_{j l}+\delta_{i l} \delta_{j k}-\frac{2}{3} \delta_{i j} \delta_{k l}\right)+t_{i j k l}+t_{i j k l}^{2}+\cdots\right)\right\rangle=\left\langle\left(\frac{\tilde{\eta}}{1-t}\right)_{i j k l}\right\rangle,
$$


with $t$ an integral operator defined by

$$
t_{i j k l}\left(\boldsymbol{r}_{1}\right)=c_{i j k l, \lambda \mu} \frac{1}{4 \pi} \int \mathrm{d} \boldsymbol{r}_{12} \frac{1}{r_{12}^{3}} Y_{i \mu}\left(\hat{r}_{12}\right) \tilde{\eta}\left(\boldsymbol{r}_{1}+\boldsymbol{r}_{12}\right),
$$

in short-hand notation

$$
t_{i j k l}=Y_{i j k l} \tilde{\eta}, \quad t_{i j k l}^{2}=Y_{i j m n} \tilde{\eta} Y_{m n k l} \tilde{\eta}, \quad \text { etc. . }
$$

Here $t_{i j k l}^{2}$ is defined analogously to $Y_{i j k l}^{2}$ given in (4.25).

The renormalized parameter $\tilde{\eta}$ is negative semi-definite if

$$
\eta_{0} \geqslant \max \left\{\eta_{1}, \eta_{2}\right\}
$$

For such a value of $\eta_{0}$ it holds

$$
\left\langle\tilde{\eta}\left(t_{i j k l}^{n}+t_{i j k l}^{n+1}\right)^{2}\right\rangle \leqslant 0, \quad n=1,2, \ldots,
$$

in which expression $t$ is the integral operator defined above in (C.2). From this inequality it follows for a statistically isotropic material that (cf. eqs. $(5.10)-(5.13))$ :

$$
T^{(2 n+1)}+2 T^{(2 n+2)}+T^{(2 n+3)} \leqslant 0 .
$$

By using this inequality for $n=1,2, \ldots$, and summing, it is deduced that

$$
\sum_{n=3}^{x} T^{(n)} \leqslant \frac{1}{2} T^{(3)}
$$

and since $T^{(3)}$ is negative semi-definite (cf. (C.4)), it follows

$$
\sum_{n=1}^{\infty} T^{(n)} \leqslant T^{(1)}+T^{(2)}
$$

Since $T^{(2)}$ vanishes for a statistically isotropic material $\left(c_{i j i j, \lambda \mu}=0\right)$, it is seen that $\eta^{*}$, given by eq. (5.9),

$$
\eta^{*}=\eta_{0} \frac{1+\frac{3}{5} \sum_{n=1}^{\infty} T^{(n)}}{1-\frac{2}{5} \Sigma_{n=1}^{\infty} T^{(n)}},
$$

possesses an upper bound, because $\eta^{*}$ is a monotonic increasing function of $\sum_{n=1}^{\infty} T^{(n)}$ :

$$
\eta^{*} \leqslant \eta_{0} \frac{1+\frac{3}{5} T^{(1)}}{1-\frac{2}{5} T^{(1)}}
$$

By choosing $\eta_{0} \leqslant \min \left\{\eta_{1}, \eta_{2}\right\}$ the renormalized parameter $\tilde{\eta}$ is positive semidefinite, from which, in the same way, a lower bound may be derived. Hence, $\eta^{*}$ is bounded by

$$
A \leqslant \eta^{*} \leqslant B,
$$


in which

$$
A=\eta_{0} \frac{1+\frac{3}{5} T^{(1)}}{1-\frac{2}{5} T^{(1)}}, \quad \text { with } \eta_{0} \leqslant \min \left\{\eta_{1}, \eta_{2}\right\}
$$

and

$$
B=\eta_{0} \frac{1+\frac{3}{5} T^{(1)}}{1-\frac{2}{5} T^{(1)}}, \quad \text { with } \eta_{0} \geqslant \max \left\{\eta_{1}, \eta_{2}\right\} .
$$

The best bounds are obtained by taking $\eta_{0}=\min \left\{\eta_{1}, \eta_{2}\right\}$ and $\eta_{0}=\max \left\{\eta_{1}, \eta_{2}\right\}$ respectively. These are the best bounds that can be indicated solely on the basis of knowledge of the values of $\eta_{1}$ and $\eta_{2}$.

\section{Appendix D}

Identification of separate terms contributing to the effective viscosity

In this appendix Schowalter et al. $^{23}$ ) are followed closely to arrive at eqs. (6.6) $-(6.9)$.

Eqs. (22) and (25) or Schowalter et al. ${ }^{23}$ ) give

$$
\begin{aligned}
& \boldsymbol{J}=c \int_{i V^{\prime+1}}\left(\left(\boldsymbol{\sigma}_{1} \cdot \boldsymbol{n}\right) \boldsymbol{r}-\eta_{1}(\boldsymbol{u n}+\boldsymbol{n u})\right) \mathrm{d} S
\end{aligned}
$$

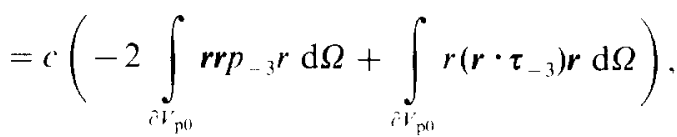

with $\boldsymbol{n}=\boldsymbol{r} / \boldsymbol{r}$ and $\mathrm{d} S=r^{2} \mathrm{~d} \Omega$.

The particles are considered as identical, independent of each other and almost spherical. The surface of a particle is indicated by $\partial V_{\text {po }}$, the number concentration by $c$ and the radius vector from the centre of the particle to a surface point by $\boldsymbol{r}$. The tensor $\tau_{-3}$ can be expressed in the solid spherical harmonic $p_{3}$ as follows:

$$
\boldsymbol{\tau}_{-3}=p_{3} \boldsymbol{1}+\frac{1}{2}\left(\boldsymbol{r} \nabla p_{-3}+\left(\boldsymbol{\nabla} p_{-3}\right) \boldsymbol{r}\right) .
$$

Eq. (24) of Schowalter et al. ${ }^{23}$ ) restricted to pure straining motion gives

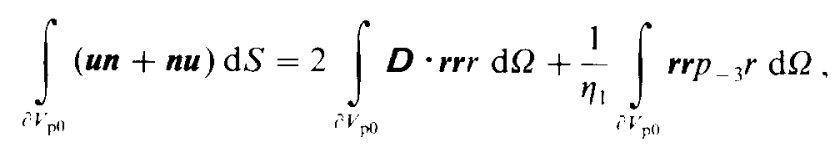

where $\boldsymbol{D}$ is the constant, traceless, symmetric rate-of-strain tensor which is applied at infinity. 
With their eqs. (26)-(28) Schowalter et al. ${ }^{23}$ ) demonstrated that

$$
\int_{\partial V_{\mathrm{p} 0}} r \boldsymbol{r} p_{-3} r \mathrm{~d} \Omega=-\frac{2}{5} \frac{1}{c} J
$$

and with neglect of the pressure term and the non-Newtonian contribution of $\boldsymbol{J}$ to the constitutive equation that

$$
J=\frac{4}{3} \pi b^{3} c 5 \eta_{1} A_{-3}^{0} D .
$$

In (D.5) $b$ is the radius of the spheres (so $\phi=\frac{4}{3} \pi b^{3} c$ ) and $A_{-3}^{0}$ is a constant typical for the flow and the emulsion.

Realizing that

$$
\int_{\partial V_{\text {р0 }}} \boldsymbol{D} \cdot \boldsymbol{r r} \mathrm{d} \Omega=\frac{4}{3} \pi b^{3} \boldsymbol{D}
$$

for spheres with radius $b$, one can conclude that

$$
\left(\eta_{2}-\eta_{1}\right) \frac{1}{V} \int_{\partial V_{\mathrm{p} 0}}(\boldsymbol{n u}+\boldsymbol{u n}) \mathrm{d} S=2\left(\eta_{2}-\eta_{1}\right)\left(1-A_{-3}^{0}\right) \phi \boldsymbol{D}
$$

and

$$
\frac{1}{V} \int_{i V_{\mathrm{p} 0}} \gamma\left(\frac{1}{3} \boldsymbol{1}-\boldsymbol{n n}\right) \mathrm{d} S=\left(A_{-3}^{0}\left(3 \eta_{1}+2 \eta_{2}\right)-2\left(\eta_{2}-\eta_{1}\right)\right) \phi \boldsymbol{D} .
$$

According to Schowalter et al. ${ }^{23}$ ) the difference between $\boldsymbol{D}$ and $\langle\boldsymbol{D}\rangle$ is on the order of $\phi$, so that $\boldsymbol{D}$ in (D.7) and (D.8) may be replaced by $\langle\boldsymbol{D}\rangle$ as long as only contributions in first order of $\phi$ are considered.

For almost spherical $(\gamma \rightarrow \infty)$ fluid particles with viscosity $\eta_{2}$ immersed in a fluid with viscosity $\eta_{1}$ subjected to pure straining motion

$$
A_{-3}^{0}=\frac{2 \eta_{1}+5 \eta_{2}}{5\left(\eta_{1}+\eta_{2}\right)}
$$

For fluid particles with viscosity $\eta_{2}$ immersed in a fluid with viscosity $\eta_{1}$ and interfacial tension $\gamma=0$ during the first moment when they are placed with a spherical form in a pure straining motion Taylor ${ }^{24}$ ) found

$$
A_{-3}^{0}=2 \frac{\eta_{2}-\eta_{1}}{3 \eta_{1}+2 \eta_{2}} .
$$

Insertion of (D.9) in (D.7) and (D.8) gives (6.6) and (6.7) respectively. Repeating this procedure with (D.10), one reproduces (6.8) and (6.9), respectively. 


\section{References}

1) J. Mewis and A.J.B. Spaull, Adv. in Colloid and Interface Sci. 6 (1976) 173.

2) R. Herczyński and I. Picńkowska, Ann. Rev. Fluid Mech. 12 (1980) 237.

3) A. Einstein, Ann. der Phys. 19 (1906) 289.

4) G.I. Taylor, Proc. Roy. Soc. Lond. A138 (1932) 41.

5) G.K. Batchelor and J.T. Green, J. Fluid Mech. 56 (1972) 401.

6) T.S. Lundgren, J. Fluid Mech. 51 (1972) 273.

7) M.J. Beran, Statistical Continuum Theories (Interscience, New York, 1968).

8) Z. Hashin, Second-Order Effects in Elasticity, Plasticity and Fluid Dynamics, M. Reiner and D. Abir, eds. (Jerusalem Academic Press, Jerusalem and Pergamon Press, Oxford, 1964) p. 434.

9) M.W.M. Willemse, Thesis, Twente University of Technology, The Netherlands (1979).

10) M.W.M. Willemse and W.J. Caspers, J. Math. Phys. 20 (1979) 1824.

11) T. Nagatani, J. Phys. Soc. Japan 47 (1979) 320

12) D. Bedeaux, R. Kapral and P. Mazur, Physica 88A (1977) 88.

13) J.B. Keller, L.A. Rubenfeld and J.E. Molyneux, J. Fluid Mech. 30 (1967) 97.

14) G.K. Batchelor, Ann. Rev. Fluid Mech. 6 (1974) 227.

15) L.D. Landau and E.M. Lifshitz, Fluid Mechanics (Pergamon, Oxford, 1959).

16) W.B. Russel, J. Rheol. 24 (1980) 287.

17) M. Oosterbroek and J. Mellema, J. Coll. Interf. Sci. 84 (1981) 14.

18) G.K. Batchelor, J. Fluid Mech. 41 (1970) 545.

19) C.E. Rosenkilde, J. Math. Phys. 8 (1967) 84.

20) E. Kröner and H. Koch, Sol. Mech. Archives 1 (1976) 183.

21) M. Hori, J. Math. Phys. 18 (1977) 487.

22) Z. Hashin and S. Shtrikman, J. Mech. Phys. Solids 11 (1963) 127.

23) W.R. Schowalter, C.E. Chaffey and H. Brenner, J. Coll. Interf. Sci. 26 (1968) 152.

24) G.I. Taylor, Proc. Roy. Soc. Lond. Al46 (1934) 501.

25) J.G. Oldroyd, Proc. Roy. Soc. Lond. A218 (1953) 122.

26) M. Oosterbrock, J. Mellema and L.S. Lopulissa, J. Coll. Interf. Sci. 84 (1981) 27.

27) J.D. Eliassen, Thesis, University of Minnesota, USA (1963). 